\title{
Prevalence of hearing impairment and outcome of universal neonatal hearing screening program in a tertiary care hospital - in UAE
}

\begin{abstract}
Background and objective: Hearing loss is one of the most common congenital anomalies, occurring in 1 to 3 per 1000 newborn infants in the well-baby nursery population, and 2 to 4 per 100 infants in the Neonatal intensive care unit graduates. The aim of the study is to identify babies with potential hearing loss and direct them to early intervention and rehabilitation. The program also initiated to create awareness about the need for detecting childhood deafness among parents and general population. An estimate of coverage, prevalence and proportion of babies defaulting newborn hearing screening program and diagnostic evaluation monitored closely to find the effectiveness of the program.

Study design: This is a retrospective study and was conducted at Latifa women and children's hospital (LWCH), a tertiary care referral hospital at Dubai, United Arab Emirates, over a period of two years from January 2018 to December 2019. Neonates admitted in Neonatal intensive care unit (NICU) and Post Natal wards (PNW) were screened. We followed three tier approach of hearing screening. All eligible neonates were subjected to Transient Evoked Oto-acoustic emission (TEOAE) prior to discharge. Automated auditory brainstem response (AABR) hearing screening was conducted in neonates who did not pass TEOAE test. Infant who failed second screening (AABR) were subjected to comprehensive hearing evaluation prior to three months of age.
\end{abstract}

Results: Out of the total 7923 neonates, $7895(99.65 \%)$ were screened and $118(1.49 \%)$ newborns referred for comprehensive audiological evaluation. Among them, 27(0.34\%) were identified with various degrees of hearing loss. The prevalence of hearing loss was found to be $0.16 \%$ and $0.92 \%$ among neonates from PNW and NICU respectively.

Conclusion: Implementation of Universal newborn hearing screening program is the need of the hour, as early detection of Hearing loss will aid early rehabilitation and better outcomes.

Keywords: hearing screen, neonates, infants, neonatal intensive care unit, preterm, hearing loss, hearing impairment
Volume II Issue 3 - 202 I

\author{
Mahmoud Saleh ElHalik,' Swarup Kumar \\ Dash, ${ }^{2}$ Arif Moinuddin Faquih, ${ }^{3}$ Rim Aref \\ Mahfouz, ${ }^{4}$ Faseela Shejee, ${ }^{5}$ Nazreen Haroon, ${ }^{5}$ \\ Khaled Mahmoud ElAtawi ${ }^{3}$ \\ 'Consultant Neonatologist \& Head of Neonatal Intensive Care \\ Unit, Latifa women and Children's Hospital, UAE \\ ${ }^{2}$ Specialist Senior Registrar Neonatologist, Neonatal intensive \\ care unit, Department of Pediatrics, Latifa women and Children's \\ Hospital, UAE \\ ${ }^{3}$ Consultant Neonatologist, Neonatal intensive care unit, \\ Department of Pediatrics, Latifa women and Children's Hospital, \\ UAE \\ ${ }^{4}$ Specialist Registrar Neonatologist, Neonatal intensive care unit, \\ Department of Pediatrics, Latifa women and Children's Hospital, \\ UAE \\ ${ }^{5}$ Audiologist, Department of Pediatrics, Latifa women and \\ Children's Hospital, UAE
}

Correspondence: Dr. Mahmoud Saleh ElHalik, Consultant Neonatologist \& Head of Neonatal Intensive Care Unit, Latifa women and Children's Hospital, Dubai, UAE, EmailmsElHalik@dha.gov.ae

Received: September 18, 2021 | Published: September 28, 2021

\section{Introduction}

Hearing loss is one of the most common congenital anomalies, occurring in 1 to 3 per 1000 newborn infants in the well-baby nursery population, and 2 to 4 per 100 infants in the Neonatal intensive care unit graduates. ${ }^{1}$ Figures from the World Health Organization estimate that about 3 per cent of people in the Middle East are affected by hearing loss. ${ }^{2,3}$ Data from the Centers for Disease Control and Prevention (CDC), United states (2016 CDC Early Hearing Detection and Identification) reported a rate of permanent hearing loss of 1.7 per 1000 infants screened for hearing loss. ${ }^{4}$ The incidence of congenital or early childhood onset sensorineural deafness or severe-to-profound hearing loss is estimated to be around $0.5-5$ in every 1000 infants as shown in studies and surveys from different countries. ${ }^{3-8}$ Over $5 \%$ (466 million people) of the world's population has disabling hearing loss (432 million adults and 34 million children). It is determined that one in ten people or over 900 million people will have hearing impairment by $2050 .^{3}$ Deafness is one of the leading health issues in the UAE and the long-term effects can be devastating.

Newborn hearing screening is done for early detection of infants with hearing loss. Normal hearing during early life is of utmost important as the first 6 months of life to 2 years of age is the critical period for the development of the auditory system and for speech and language acquisition. ${ }^{9,10}$ Hearing impairment leads to sensory deprivation which, affects infant's perception of speech sounds and their capacity to learn..$^{9-11}$ This key fact made it necessary to identify hearing challenged during the early years of life. It is recommended that all infant in the post-natal wards to be screened for hearing loss prior to 1 month of age and NICU infants to be screened near to discharge or when medically stable. ${ }^{12}$

Tests used for newborns hearing screening include Transient Evoked Oto-acoustic Emissions (TEOAE) and Automated Brainstem Evoked Response Audiometry (AABR). Both of these technologies are noninvasive and can be easily performed in neonates and infant. ${ }^{13}$ 
TEOAE screening is highly sensitive (between $85 \%$ and $100 \%$ ) and reasonably specific (between $91 \%$ and $95 \%$ ). ${ }^{14}$ AABR is also highly sensitive and specific (90-95\%), and has the additional advantage of identifying newborns with auditory neuropathy unlike TEOAE testing. ${ }^{15,16}$

In the three step screening methods, infants who do not pass the initial TEOAE screening are subjected to repeat TEOAE and AABR (if TEOAE failed) test after 2 weeks. If AABR is not passed the infant referred immediately for a comprehensive audiological evaluation, with a goal of having hearing loss confirmed by 3 months of age and appropriate early intervention should be done by 6 months of age. Joint committee of Infant hearing has recommended to have at least one comprehensive Audiological evaluation by 9 months of age or before, based on the risk factors of late onset hearing loss. ${ }^{17}$ It is important to screen newborns irrespective of risk factors which helps in better identification of hearing loss and helps in initiating early intervention and habilitation during the critical period. Evidence shows that, average age at diagnosis of hearing disorders was 4.6 months for children who are screened and 34.9 months for children who were not screened. ${ }^{18}$

\section{Aims and objectives of the study}

We conducted this study to evaluate the coverage, prevalence, initial screening referral rate, diagnostic referral rate and the number of defaulters (infants who failed to return for follow-up screening and those who missed diagnostic evaluation) and to create awareness about the need for detecting childhood deafness among parents and general population.

Primary objective:

To identify infants with potential hearing loss early and direct them for early intervention and rehabilitation.

Secondary objectives:

- $\quad$ To cover $95 \%$ of babies born in LWCH for newborn hearing screening.

To identify the percentage of babies defaulting newborn hearing screening.

\section{Material and methods}

This is a retrospective study, which was conducted at Latifa women and children's hospital, a tertiary care referral hospital at Dubai, United Arab Emirates, over a period of two years from January 2018 to December 2019. Newborns who were admitted to post-natal wards (PNW) and neonatal intensive care units (NICU) during the study period were included. Infants with external ear abnormalities or other abnormalities which would affect the placement of transducers or electrodes to carry out the testing procedure and babies transferred out to other hospitals were excluded.

Hearing Screening is done as routine medical service for all neonates with the verbal consent of the parents. Neonates in PNW were screened preferably after $24 \mathrm{hrs}$ hours of age or near to discharge to avoid the higher referral rate which might occur due to residual birthing debris in the ear canal. NICU infants were screened as close to discharge and/or when they are medically stable. The screening protocol involves three stages, 2 stages of screening and a diagnostic Audiological evaluation if required. The initial screening was done at the bedside by utilizing TEOAE. Calibration is automatically performed prior to the test and TEOAEs recorded automatically. At the completion of the test, a Pass or Refer result was displayed. Results are recorded in the electronic medical records after completion of the procedure. Infants with refer in the initial screening is rescreened after two weeks using same TEOAE in the outpatient clinic. If during rescreening, TEOAE failed, further screening with AABR done in the same session. Infants who did not fulfill the pass criteria after the AABR test were referred for diagnostic audiological assessment to confirm the existence of hearing loss primarily by diagnostic ABR/ BERA (Brainstem Evoked Response Audiometry). The ABR results were interpreted either as normal, auditory maturation delay or as hearing loss of various severity and type. Those neonates interpreted with auditory maturation delay were further evaluated at 3 months to rule out delayed maturation of auditory pathway. Parents were asked to monitor auditory milestones and were advised to return to the facility if any delay in attaining milestones were observed.

Descriptive analyses were used with results presented as proportions. Data were analyzed using Microsoft excel 2016 and online calculators. The study was approved by Dubai Scientific Research Ethics Committee (DSREC-06/2021_12).

\section{Results}

Amongst the total eligible babies $(\mathrm{n}=7923)$ born in Latifa women and children Hospital, 7895 (99.65\%) infants were screened, and $28(0.35 \%)$ neonates defaulted initial hearing screening. A total of $7185(91.10 \%)$ passed first screening and 710 infants $(9.90 \%)$ failed first screening. Out of the total 710 babies scheduled for second screening, 529(74.51\%) attended the second screening and $181(25.49 \%)$ were lost to follow-up. Amongst the neonates who attended the second screening, 411(77.69\%) passed and $118(22.31 \%)$, which represent $1.49 \%$ of all screened infants $(\mathrm{N}=7895)$ were referred for comprehensive audiological evaluation by Otolaryngologists. Out of the 118 referred infants, $45(38.14 \%)$ were from PNW and 73 $(61.86 \%)$ were from NICU (Flow chart $1 \& 2$ ) (Table 1).

Out of $118(22.31 \%)$ babies referred, $76(64.40 \%)$ attended diagnostic evaluation. Among them 49(64.48\%) babies were identified with normal hearing sensitivity and 27(35.52\%) babies were identified with various degrees of hearing loss, which accounts to $0.34 \%$ of total eligible infants. Forty two $(35.60 \%)$ neonates were lost to follow up. Prevalence of hearing loss in NICU was $17(0.92 \%)$ and in postnatal ward were $10(0.16 \%)$. Eleven infants $(0.13 \%$ of total screened) were identified with sensorineural hearing loss. Among whom 10 were having severe to profound hearing loss (5 of which were having unilateral hearing loss) and 1 with moderate hearing loss. Sixteen $(0.20 \%$ of total screened) were identified with mild to moderate degree of conductive hearing loss. Three infants were fitted with cochlear implant, and one was fitted with hearing aid. The infants identified with conductive hearing loss were advised follow up for medical evaluation and intervention.

Table I Prevalence of hearing loss

\begin{tabular}{lll}
\hline Prevalence of hearing loss & NICU & PNW \\
\hline Total babies screened & 1847 & 6048 \\
Babies with hearing loss & 17 & 10 \\
Prevalence & $0.92 \%$ & $0.16 \%$ \\
\hline
\end{tabular}




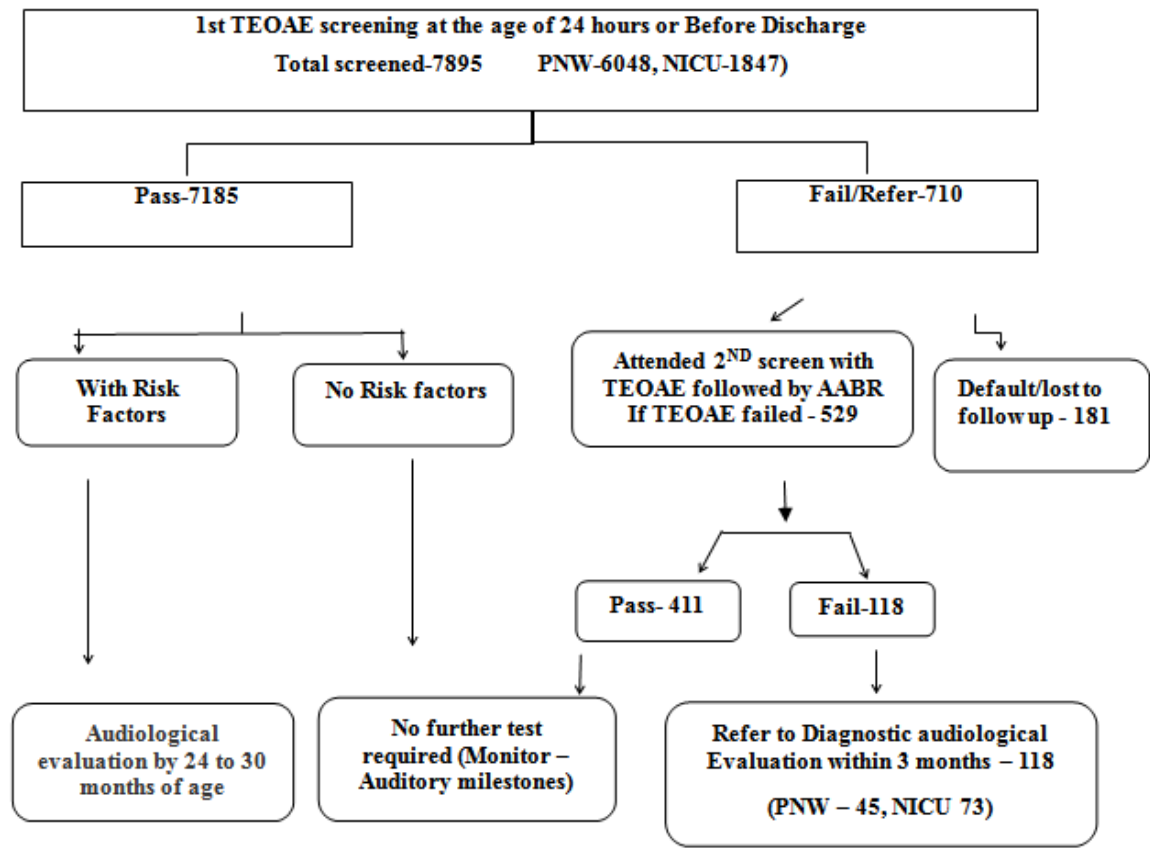

Flowchart I Flow of infants subjected to hearing screening.

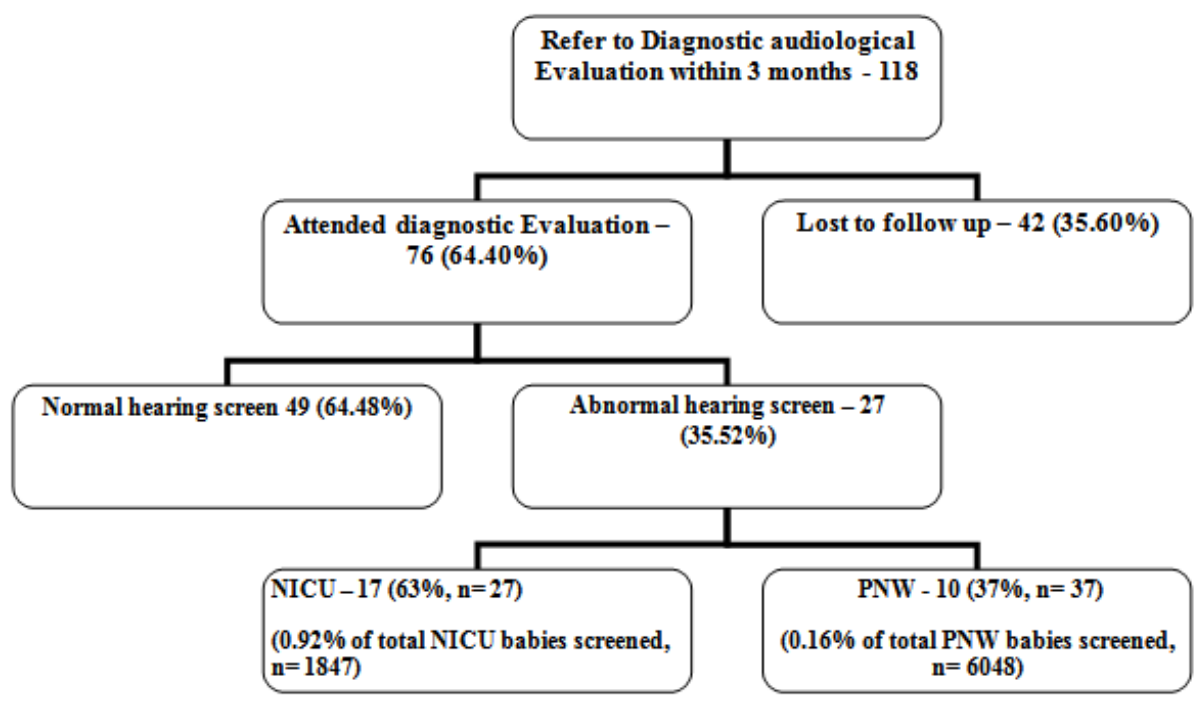

Flowchart 2 Prevalence of hearing impairment.

\section{Discussion}

Congenital hearing loss is one of the most common and major abnormalities present at birth, which needs to be diagnosed early, inorder to prevent problems related to speech, language, and cognitive development. Early and prompt detection of hearing abnormality and relevant intervention can greatly reduce its adverse effect on development. The number of neonates screened for initial TEOAE hearing test varies from $84 \%-98 \%$ in different studies. ${ }^{6,8}$ In our study the rate was $99.65 \%$, which is within the quality indicator of screening established by JCIH (i.e. $>95 \%) .{ }^{17}$ In our institution, hearing screening service is available throughout the week to attain maximum coverage.

First and second screening is completed within first month of age in most of the eligible infants. Out of the screened infants, $9.90 \%$ were referred for the $2^{\text {nd }}$ screening, which is comparable to findings in other studies. ${ }^{5,6}$ Lost to follow-up for $2^{\text {nd }}$ screening was $25.49 \%$, which was less than the default rate (33.9\%) seen by Amirozi A. et al. ${ }^{8}$ High default rates would affect the quality and effectiveness of the program as it reduces the detection of newborns with hearing loss. Mukari et al. found that four factors contributed to the high Default rates like poor follow-up protocol, lack of communication between the parents and screening personnel, lack of parent awareness regarding hearing loss and the need for early intervention, and problems in transportation. ${ }^{19}$

We found that $1.49 \%$ of total eligible infants was referred for detailed audiological assessment. Referral rates are within the program quality standard target of $4 \% .{ }^{17}$ After diagnostic audiological evaluation, $27(35.52 \%)$ infants $(0.34 \%$ of all eligible infants) were identified with various degrees of hearing loss. Out of which, 17 infants were from NICU and 10 from PNW. Prevalence of hearing loss among infants from NICU was $0.92 \%$, which was significantly higher than the prevalence of $0.16 \%$ observed in PNW infants. Our findings were similar to results of other studies. ${ }^{7,26}$ 
The universal newborn hearing screening (UNHS) program is designed to identify babies who have hearing loss and to provide necessary intervention as soon as possible. Dependence on physician observation and parental identification has not been successful in the past in detecting significant hearing loss in the 1st year of life. An approach to perform hearing screening only in high risk infants having risk factor for hearing loss may miss up to $50 \%$ of hearing impairment. 19,21,23,24 These factors have resulted in an average age of identification of 11-19 months for children with known risk factors for hearing loss and 15-19 months for children without apparent risk. $^{20,22,24}$ In about 50 percent of newborns with permanent bilateral congenital hearing loss, no identifiable risk factors have been found. In the presence of a structured program for universal hearing screening (irrespective of risk factors); age of identification of hearing loss and intervention can be lowered, which positively affect the long-term development of the child. The success of a newborn hearing screening program depends on many components, such as dedication from various disciplines, constant rating of the program and determined attempts towards raising public awareness about hearing loss and early intervention. Newborn hearing screening program still needs constant evaluation to improve the quality of the program.

Strengths of our study are, implementation of universal hearing screening irrespective of presence or absence of risk factors, multistage screening approach and proper follow up plan. Our data collection methods were robust. Our main limitations are, retrospective design, many infants are lost to follow up. It would have been better to use AABR in place of TEOAE as first screening methods for high risk NICU graduates.

\section{Conclusion}

The overall prevalence of hearing loss in Latifa Women and Children Hospital was found to be $0.34 \%$. Infants who were graduated from NICU were having higher rate of hearing loss $0.92 \%$, compared to PNW babies, $0.16 \%$. Hearing loss is one of the commonest curable disabilities in children, even in babies without any risk factors. Newborn hearing screening is especially important in identifying early hearing Impairment leading to early intervention, which may leads to better quality of life. So, every institution should have a universal hearing screening program based on multistage approach.

\section{Acknowledgments}

Thanks all the parents of the infants who were part of the study. Acknowledge the co-authors for their help and support.

\section{Conflicts of interest}

None.

\section{Funding}

None.

\section{References}

1. American Academy of Pediatrics, Newborn and infant Hearing loss, Detection, and intervention Task force on Newborn and infant Hearing, Pediatrics. 1999;103:527-530.

2. Deafness and Hearing loss. World Health Organization.

3. WHO global estimates on prevalence of hearing loss; prevention of deafness WHO, 2018.

4. Summary of 2016 National CDC EHDI Data.
5. Satish HS, Anil Kumar R, Viswanatha B. Screening of Newborn Hearing at a Tertiary Care Hospital in South India. Indian Journal of Otolaryngology Head Neck Surg. 2019;71(Suppl 2):1383-1390.

6. Habib HS, Abdelgaffar H. Neonatal hearing screening with transient evoked otoacoustic emissions in Western Saudi Arabia. International Journal of Pediatric Otorhinolaryngology. 2005;69(6):839-842.

7. Kavita Sachdeva, Tulisi Sao. Outcomes of Newborn Hearing Screening Program: A Hospital Based Study. Indian Journal of Otolaryngology Head Neck Surgery. 2017;69(2):194-198.

8. Amirozi Ahmad, Irfan Mohamad, Suzana Mansor, Mohd Khairi Daud and Dinsuhaimi Sidek, Outcome of a newborn hearing screening program in a tertiary hospital in Malaysia: the first five years, published Online: 2011.

9. J.L. Northern, M.P. Downs, Hearing in Children, fourth ed. Williams \& Wilkins, Baltimore, MD, 1991, pp.1-31.

10. Steol-Gammon C, Otomo K. Babbling development of hearing -impaired and normally hearing subjects. J Speech Hear Disorderr. 1986:51:33-41.

11. Elliott LL, Armbruster VB. Some possible effects of the delay of early treatment of deafness. J speech Hear Res. 1967;10:209-224.

12. Year 2007 Position Statement: Principles and Guidelines for Early Hearing Detection and Intervention Programs. Joint Committee on Infant Hearing, Pediatrics. 2007;120;8988.

13. Norton SJ, Gorga MP, Widen JE, et al. Identification of neonatal hearing impairment: evaluation of transient evoked otoacoustic emission, distortion product otoacoustic emission, and auditory brain stem response test performance. Ear Hear. 2000;21:508-528.

14. Janssen T. A review of the effectiveness of otoacoustic emissions for evaluating hearing status after newborn screening. Otol Neurotol. 2013;34:1058-1063.

15. Katarzyna E. Wroblewska-Seniuk, Piotr Dabrowski, Witold Szyfter and Jan Mazela; Universal newborn hearing screening: methods and results, obstacles, and benefits. Pediatric Research. 2017;81(3).

16. Saeed Heidari, Alireza Olyaee Manesh, Fatemeh Rajabi. The sensitivity and specificity of automated auditory brainstem response and otoacoustic emission in neonatal hearing screening: a systematic review. Aud Vest Res. 2015;24(3):141-15.

17. Year 2019 Position Statement: Principles and Guidelines for Early Hearing Detection and Intervention Programs, The Joint Committee on Infant Hearing; The Journal of Early Hearing Detection and Intervention 2019; 4(2)

18. Katrin Neumann, Harald A, Karl R. "A Survey on the Global Status of Newborn and Infant Hearing Screening”. The Journal of Early Hearing Detection and Intervention. 2000;2(2):63-84.

19. Mukari SZ, Tan KY, Abdullah A. A pilot project on hospital-based universal newborn hearing screening: Lessons learned. Int J Pediatr Otorhinolaryngol. 2006;70:843-851.

20. Mauk GW, Behrens TR. Historical, political, and technological context associated with early identification of hearing loss. Semin Hear. $1993 ; 14: 1-17$.

21. Parving A. Congenital hearing disability: epidemiology and identification: a comparison between two health authority districts. Int $J$ Pediatr Otolaryngol. 1993;27:29-46.

22. Watkins $\mathrm{P}$, Baldwin M, McEnery G. Neonatal at risk screening and the identification of deafness. Arch Dis Child. 1991;66:1130-1135.

23. Elssmann S, Matkin N, Sabo M. Early identification of congenital sensorineural hearing impairment. Hear J. 1987;40:13-17.

24. Pappas DG. A study of the high-risk registry for sensorineural hearing loss. Arch Otolaryngol Head Neck Surg. 1983;91:41-44. 
25. Stein L. On the real age of identification of congenital hearing loss. Audiol Today 7:10-11 Harrison M, Roush J. Age of suspicion, identification, and intervention for infants and young children with hearing loss: a national study. Ear Hear. 1996;17:55-62.
26. M Antoni, I Rouillon, F Denoyelle, et al. Newborn hearing screening: Prevalence and medical and paramedical treatment of bilateral hearing loss in a neonatal series in the Île-de-France region of France. European Annals of Otorhinolaryngology, Head and Neck diseases. 2016;133:9599. 